\title{
Analysis of Chloroplast Genome of Two Cytoplasmic Male Sterile Lines Derived from Interspecific Chimera and Intergeneric Somatic Hybrid in Brassicaceae
}

\author{
Yuzo Ohta ${ }^{1)}$, Yutaka Hirata ${ }^{* 2)}$, Takeshi Motegi ${ }^{2)}$, Gen Hattori ${ }^{1)}$ and Takashi Noguchi ${ }^{2)}$ \\ 1) United Graduate School of Agriculture, Tokyo University of Agriculture and Technology, 3-5-8 Saiwai, Fuchu, Tokyo 183-8509, Japan \\ 2) Graduate School of Agriculture, Tokyo University of Agriculture and Technology, 3-5-8 Saiwai, Fuchu, Tokyo 183-8509, Japan
}

\begin{abstract}
Two new types of cytoplasmic male sterile (CMS) lines have been developed, one by in vitro chimera formation between Brassica rapa and B. oleracea and another by protoplast fusion of B. oleracea with Raphanus sativus. These CMS lines harbored an Ogura-type mitochondrial gene, orf138. They expressed chlorosis at a young stage and their chloroplast genome changed to the type of Ogura radish based on restriction fragment length polymorphism (RFLP) analysis. Thus, we attempted to study the relationships among cytoplasmic male sterility, chlorosis and the variation of the chloroplast genome using the matK and RuBisCO genes. The two CMS lines, normal and male sterile radishes exhibited similar mat $K$ gene sequences, while $B$. rapa and $B$. oleracea showed matK sequences of the radish type in very low copy numbers. The CMS lines displayed an identical RFLP pattern with that of the male sterile radish in the RuBisCO gene region. These phenomena indicated that the chloroplast genome organization had changed from Brassica to Raphanus type or Raphanus CMS type by CMS induction, and suggested the existence of heteroplasia in the Brassica chloroplast.
\end{abstract}

Key Words: Cytoplasmic male sterility, interspecific Brassica chimera, chloroplast, matK.

\section{Introduction}

We developed two new cytoplasmic male sterility (CMS) lines, one derived from the interspecific Brassica chimera between 'Komatsuna' (B. rapa) and red cabbage, 'Ruby Ball' (B. oleracea) (Noguchi et al. 1992, Noguchi and Hirata 1994, Motegi et al. 2003), and another from the intergeneric somatic hybrid between 'Shogoin' (R. sativus) and 'Ruby Ball' (Kameya et al. 1989, Motegi et al. 2003). Molecular analysis of the mitochondrial genome was performed to elucidate the induction mechanism of cytoplasmic male sterility (male sterility, hereafter) (Motegi et al. 2003). These CMS lines harbored the Ogura-specific orf 138 gene, responsible for radish CMS (Makaroff et al. 1991, Yamagishi and Terachi 2001). The CMS lines were very stable without anthers or pollen, i.e., fertility was not recovered under fluctuating conditions.

These two new types of CMS lines are suitable plant materials for studying the CMS regulation system. Southern blot analysis of the chimera-derived CMS line using 16 mitochondrial probes revealed band patterns similar to that of the 'Kosena' radish mitochondrial genome (Hirata et al. 2000). In addition, the somatic hybrid between normal fer-

Communicated by H. Yamagishi

Received July 23, 2005. Accepted December 6, 2005.

*Corresponding author (e-mail: yhirata@cc.tuat.ac.jp) tile radish and normal fertile cabbage clearly maintained a genome with a small amount of Ogura type cytoplasm (Kameya et al. 1989, Motegi 1999).

Previously, we analyzed these CMS lines using the mitochondrial Ogura-specific atp6 (Makaroff et al. 1989) and orf138 genes (Makaroff et al. 1991). The chimera-derived CMS, protoplast fusion-derived CMS and radish with Ogura CMS displayed a similar genome structure (Hirata et al. 2000, Motegi et al. 2003). Interestingly, these two new CMS types showed similar RFLP patterns in the mitochondria and chloroplast, although their origin was different. Furthermore, these CMS lines expressed chlorosis at the seedling stage. It is generally recognized that Brassica CMS lines express chlorosis (Ohkawa 1986) and that the chloroplast genome participates in the expression of chlorosis, while the interrelationship between the CMS and chloroplast has not been fully elucidated or was ruled out (Ohkawa 1986). However, because of the similarity of the changes in the mitochondrial and chloroplast RFLP patterns to those of the Ogura CMS type, we analyzed the organization of the chloroplast genome compared with that of the mitochondrial genome (Motegi et al. 2003). Motegi (1999) performed a Southern blot analysis using BamHI-digested BAC clones from the rice chloroplast genome, because the BAC clones were constructed in rice at that time (Hirai et al. 1985). As a result, differences in the RFLP patterns between fertile radish and a CMS radish were revealed. 
In order to gain a better understanding of the structure of the chloroplast genome in the CMS lines, we performed an analysis of the chloroplast genome in the region that herbored the matK and RuBisCO genes. The matK gene in the chloroplast codes for the intron within the $\operatorname{trnK}$ gene (Neuhaus and Link 1987, Liere and Link 1995, Ostheimer et al. 2003). Classification and systematics analyses have been widely performed at the generic and specific levels using the specific matK sequence polymorphism (Steele and Vilgalys 1994, Soltis et al. 1996, Xiang et al. 1998, Wu et al. 2000, Koch et al. 2001). Both of the CMS lines displayed a structure similar to that of radish in matK gene region, while, they exhibited a RFLP pattern specific to that of the male sterile radish of the $\mathrm{RuBisCO}$ gene site.

\section{Materials and Methods}

\section{Plant materials}

Our laboratory has been maintaining cytoplasmic male sterile (CMS) lines originating from chimeras obtained by interspecific in vitro grafting between 'Komatsuna' (Brassica rapa) and 'Ruby Ball' (B. oleracea) (Noguchi et al. 1992). These CMS lines were designated as chimera-derivedCMS (CDC) lines. On the other hand, the CMS cabbage, an intergeneric somatic hybrid, was synthesized by protoplast fusion between B. oleracea cv. 'Ruby Ball' and Raphanus sativus cv. 'Shogoin' by Kameya et al. (1989). These CMS lines were designated as protoplast-fusion-derived CMS (PDC) lines. We used these two types of CMS lines and respective parental plants in the present study (Hirata et al. 2000, Motegi et al. 2003). As a comparable standard, the Ogura-type CMS cv. 'MS-Gensuke' (R. sativus) was adopted (Ogura 1968, Makaroff et al. 1991, Yamagishi and Terachi 2001). Normal fertile radish 'Uchiki-Gensuke' was used as a maintainer for 'MS-Gensuke', while 'Comet' (R. sativus) as a restorer of Ogura CMS. The B. rapa Noko (N) line was also used for the RFLP analysis.

\section{DNA extraction and primer design}

Total DNA was extracted from young leaves by the modified CTAB method (Aldrich and Cullis 1993). MatK was amplified by the polymerase chain reaction (PCR) method with $m a t K$ gene-specific sequences as primers. The primers were designed from the complete chloroplast sequences based on the clustal W analysis in Oryza sativa (NC 001320), Zea mays (NC 001666), Arabidopsis thaliana (NC 001879), Nicotiana tabacum (NC 000932) and Spinacia oleracea (NC 002202) chloroplasts.

\section{PCR amplification and TA cloning}

PCR amplification was performed in a $25 \mu \mathrm{l}$ reaction solution containing $16.87 \mu \mathrm{l}$ of sterile water, $2.5 \mu \mathrm{l}$ of $10 \times$ Taq polymerase reaction buffer (Takara Bio Co. Ltd. Otsu, Shiga, Japan), $2.0 \mu \mathrm{l}$ of $2.5 \mathrm{mM}$ dNTPs in an equimolar ratio, $0.13 \mu \mathrm{l}$ of Taq DNA polymerase ( 5 unit/ $\mu \mathrm{l}$ ) (Takara Bio Co. Ltd.), $1.0 \mu \mathrm{l}$ of $12.5 \mu \mathrm{M}$ matK gene-specific cpp02 (5'-CGC
GTC TCT CTA AAA TTG CAG-3') and cpp03 (5'-ATC WAT TCA TTC AAY ATT TCC-3') primers (Fig. 1), $1.5 \mu \mathrm{l}$ of $25 \mathrm{mM} \mathrm{MgCl}_{2}$ and $1.0 \mu \mathrm{l}$ of template DNA $(25 \mathrm{ng} / \mu \mathrm{l})$. The solutions were first denatured for $2 \mathrm{~min}$ at $94^{\circ} \mathrm{C}$, followed by 30 cycles of amplification consisting of $30 \mathrm{sec}$ at $94^{\circ} \mathrm{C}$ (denaturation), $30 \mathrm{sec}$ at $50^{\circ} \mathrm{C}$ (annealing), and $2 \mathrm{~min}$ at $72^{\circ} \mathrm{C}$ (polymerization). After amplification, the products were ligated into pT7blue vectors (Novagen Co. Madison, WI, USA) and used for DNA sequencing analysis.

The purified plasmid DNA of each clone was sequenced on the ABI PRISM 377 DNA sequencer (Applied Biosystems, Foster, CA, USA) using the DYEnamic ET terminator cycle sequencing kit (Amersham Biosciences, Piscataway, NJ, USA). DNA sequences were aligned manually by using GENETIX-WIN 4.0 (GENETYX Co. Tokyo, Japan). The BLAST and the CLUSTAL W programs were used for searching the homology among nucleotide sequences. PCR primer was designed from a sequence specific to 'CDC', 'PDC', 'Shogoin' and 'MS-Gensuke' matK gene, and was designated as Radish sp (5'-ATC TTA GTC TTA CTG CTT ACT G-3'). PCR was conducted with the primer pair of Radish sp and cpp03 (Fig. 1). PCR was performed by the above method with 30 and/or 45 cycles.

Furthermore, a new specific marker that can amplify the $8 \mathrm{~kb}$ region present far from the mat $K$ gene region was designed to investigate RFLP between the CMS and the fertile one. The region included the $\mathrm{RuBisCO}$ gene part on the data base search (abbreviated as RuBisCO gene sequence). PCR primers were designed from a conserved sequence in 'CDC', 'PDC' and 'MS-Gensuke'. The forward (5'-GGA ATC TTC TAC TGG TAC ATG GAC AAC TGT-3') and the reverse (5'-GGC TAA ATG GCA ATT GGC ACA TAC AAT $-3^{\prime}$ ) primers were used for PCR amplification. PCR was performed using KOD plus DNA polymerase (Toyobo Co., Ltd. Osaka, Osaka, Japan). The solutions were firstly denatured for $2 \mathrm{~min}$ at $94^{\circ} \mathrm{C}$, followed by 30 cycles of amplification consisting of $20 \mathrm{sec}$ at $94^{\circ} \mathrm{C}$ (denaturation), $8 \mathrm{~min}$ at $68^{\circ} \mathrm{C}$ (annealing of primers and polymerization). The PCR product was used to synthesize the probe for CDC amplification.

\section{Southern blot analysis}

Southern blot analysis was performed by a conventional method (Roche Diagnostics, Mannheim, Germany; Tateishi et al. 2001, 2002). Genomic DNA (10 $\mu \mathrm{g})$ was digested with the MspI restriction enzyme. Digested DNAs were separated by electrophoresis on $0.8 \%$ TAE-agarose gel and transferred onto a Hybond $\mathrm{N}+$ membrane (Amersham Biosciences). The DIG-labeled matK gene PCR product and RuBisCO gene PCR product were used as hybridization probes. The membrane was washed once at $68^{\circ} \mathrm{C}$ for $15 \mathrm{~min}$ after hybridization.

\section{Results and Discussion}

We amplified the matK gene from the CMS lines and 
fertile parental lines using the cpp02 and cpp03 primers (Fig. 1). Based on the comparison of the sequencing data, the PCR products were found to show almost the same size as follows : $1,765 \mathrm{bp}$ in 'CDC', 1,762 bp in 'PDC', 1,758 bp in 'Ruby Ball', 1,756 bp in 'Komatsuna', 1,765 bp in 'Shogoin' and 1,764 bp in 'MS-Gensuke'. These sequences corresponded to those of the matK genes based on BLAST complementation (Koch et al. 2001). 'CDC' and 'PDC', the CMS radish and fertile 'Shogoin' displayed a common seven-base pair region that was not found in 'Ruby Ball' and 'Komatsuna' (Fig. 2).

Using the specific primers (Radish sp and cpp03), the band was amplified by PCR with 30 cycles in the CMS lines and fertile radish, but not in 'Ruby Ball' or 'Komatsuna'. However, 'Ruby Ball' and 'Komatsuna' showed a similar molecular size of band by PCR with 45 cycles (Fig. 3 ). These amplified bands obtained with 45 cycles were cloned into the pT7 blue vector and sequenced, showing almost the same size, namely 1,271 bp in 'CDC', 'PDC', 'Ruby Ball', and 'Komatsuna', as well as 1,270 bp in 'Shogoin' and 1,272 bp in 'MS-Gensuke' (Fig. 2 and Fig. 3). In addition, the $M s p$ I restriction site was also found at a Radish-specific position in the sequence. The fertile line had two MspI restriction sites and the CMS line had three sites in the matK sequence (Fig. 1, Fig. 2 and Fig. 3).
Although no PCR products were obtained in the fertile Brassica plants with the Radish sp primer after the conventional 30 cycles, a faint band was observed with 45 cycles, suggesting the existence of heteroplastic molecules like in the case of mitochondrial orf138. Our findings indicated the presence of two different molecular species with different copy numbers. This heteroplastic phenomenon has been well documented in the case of the mitochondrial orf138 and related genes (Makaroff et al. 1991). In the previous experiment, three molecules were observed and the copy numbers varied (1,000 times difference) between the CMS lines and

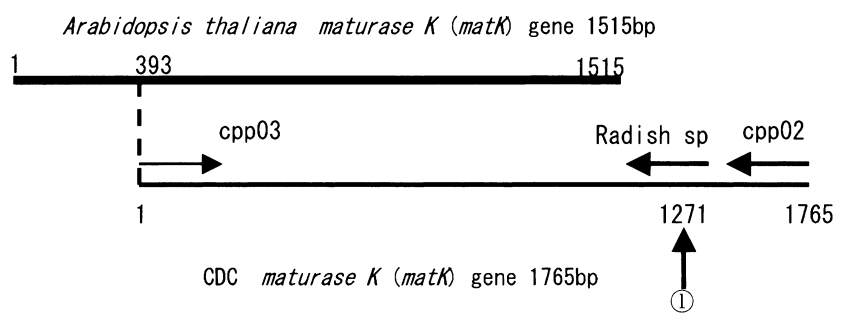

Fig. 1. Primer positions in the matK region for identification of the polymorphism in 'CDC' and Arabidopsis thaliana. MatK gene regions of ' $\mathrm{CDC}$ ' and Arabidopsis thaliana are comparatively shown. Designed primers cpp02, cpp03 and Radish sp are indicated by arrows. (1) corresponds to the site lacking seven bases.

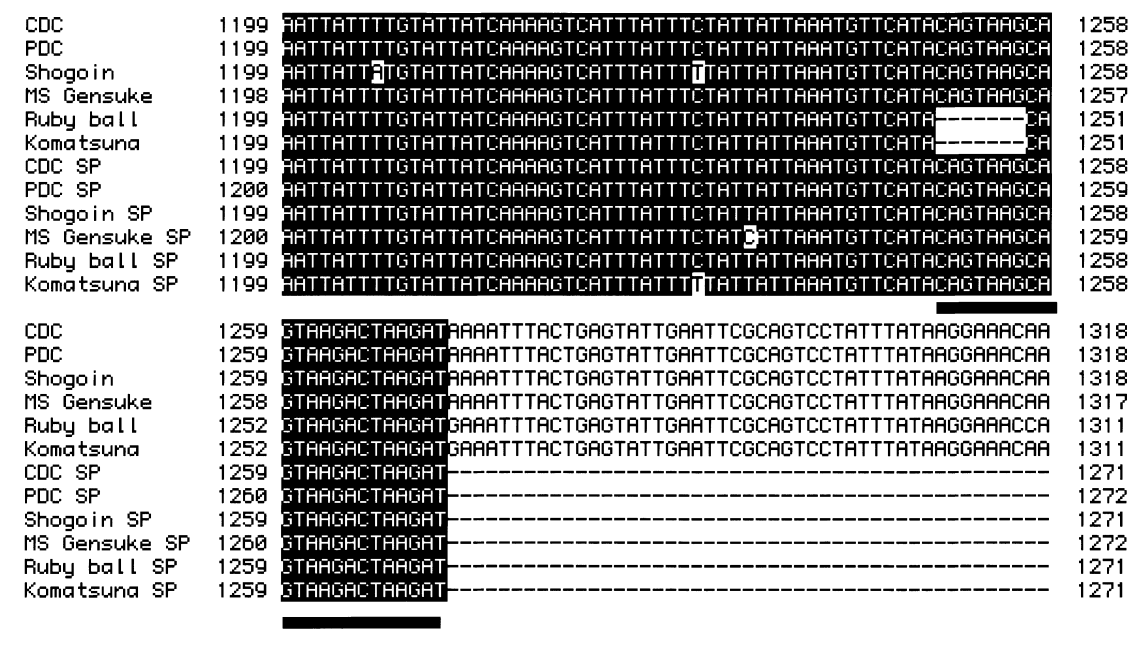

Fig. 2. Comparison of matK sequences between CMS lines and fertile lines. 'CDC' sp, 'PDC' sp, 'Ruby Ball' sp, 'Komatsuna' sp, 'Shogoin' sp and 'MS-Gensuke' sp denote the radish specific sequence, respectively. The Radish sp primer site is underlined.

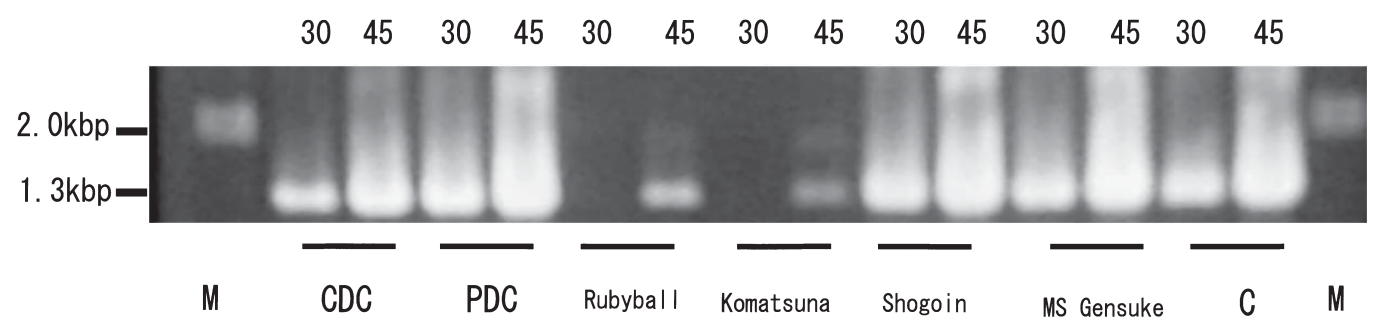

Fig. 3. PCR analysis using the Radish sp primer and cpp03 primer with 30 and 45 cycles. 'CDC', 'PDC', C and M denote chimera-derived CMS, protoplast fusion-derived CMS, restorer radish cv. 'Comet', and molecular size marker $(2.0 \mathrm{kbp})$. 
fertile mitochondria (Motegi et al. 2003).

The RFLP pattern of the CMS lines in the matK region was similar to that of fertile radish and different from that of fertile B. rapa and B. oleracea (Fig. 4). However, bands with a similar size to that of 'CDC', 'PDC', 'Shogoin' and 'MSGensuke' were also observed in 'Komatsuna' and 'Ruby Ball', though the latter two varieties showed very faint bands. On the other hand, 'PDC', which is the asymmetric somatic hybrid between 'Ruby Ball' and 'Shogoin', exhibited molecules specific to both parents. We found that the matK gene in ' $\mathrm{CDC}$ ' had a homologous sequence to that of radish. Interestingly, the results suggested that in fertile Brassica, the radish type matK gene was present in very low copy numbers. The causal mechanism of the shift of the mat $K$ gene structure of the CMS lines to the radish type has not been determined and further studies should be carried out (Fig. 4 and Fig. 5).

The results obtained by Southern blot analysis using the $\mathrm{RuBisCO}$ probe were different from those of the RFLP analysis in the matK gene region (Fig. 5). Amplification bands with the RuBisCO gene primer could identify differences among fertile Brassica and the radish parents, the CMS lines and CMS radish. Differences in the RFLP pattern between the fertile parents and CMS lines ('CDC', 'PDC' and 'MSGensuke') were observed. The pattern of the CMS lines was different from that of the radish variety, 'Shogoin' with normal cytoplasm. The results indicated that the CMS lines showed a different RuBisCO structure from that of the fertile lines, suggesting some relation with the expression of chlorosis in the CMS lines.

The results of this experiment suggested that a stoichiometric shift might have occurred in the chloroplast genome of the two CMS lines by a still unknown mechanism. The analysis of the cytoplasmic gene structure in more detail might shed light on the organelle genome structure and the CMS regulation mechanism(s) of the two lines.

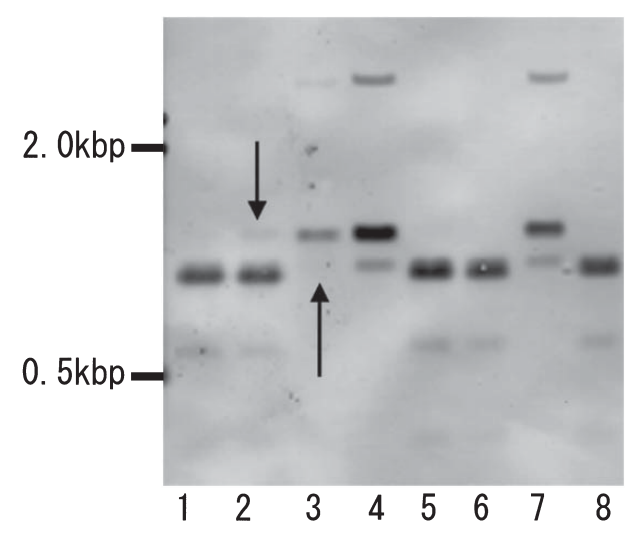

Fig. 4. Southern blot analysis of CMS lines and fertile plants probed with the PCR product of the matK gene in CDC line. Genomic DNA of each material was digested with $M s p$ I. The arrow indicates the minor bands. 1, 'CDC'; 2, 'PDC'; 3, 'Ruby Ball'; 4, 'Komatsuna'; 5, 'Shougoin'; 6, 'MS-Gensuke'; 7, 'N line'; 8, 'Comet'.

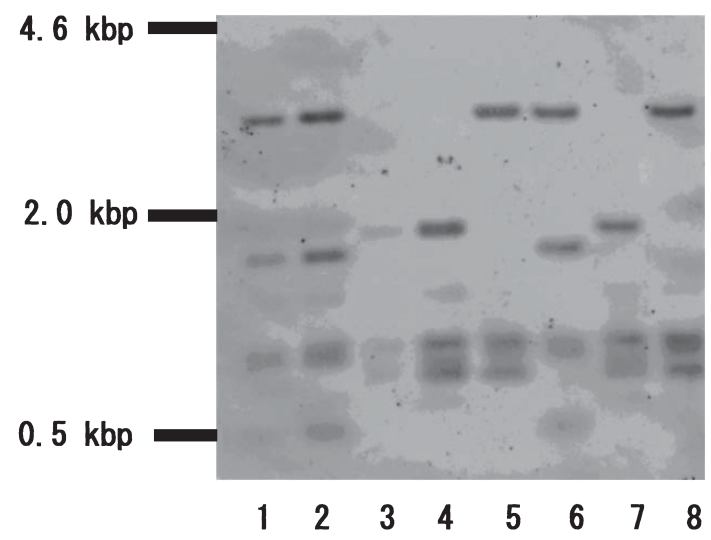

Fig. 5. Southern blot analysis of CMS lines and fertile plants probed with the PCR product of the RuBisCO gene in the CDC line. Genomic DNA of each material was digested with $M s p$ I. 1, 'CDC'; 2, 'PDC'; 3, 'Ruby Ball'; 4, 'Komatsuna'; 5, 'Shougoin'; 6, 'MS-Gensuke'; 7, 'N line'; 8, 'Comet'.

\section{Literature Cited}

Aldrich,J. and C.A.Cullis (1993) RAPD analysis in flax: Optimization of yield and reproducibility using KlenTaq1 DNA polymerase, Chelex 100, and gel purification of genomic DNA. Plant Mol. Biol. Rep. 11: 128-141.

Hirai, A., T. Ishibashi，A. Mriyuki， N. Iwatsuki，K. Shinozaki and M.Sugiura (1985) Rice chloroplast DNA: a physical map and the location of the genes for the large subunit of ribulose 1, 5bisphosphate carboxylase and 32KD photosystem II reaction center protein. Theor. Appl. Genet. 70: 117-122.

Hirata,Y., T.Motegi, Y.Takeda and K.Morikawa (2000) Induction of cytoplasmic male sterility in the seed progeny derived from artificially-synthesized interspecific chimera in Brassica. Euphytica 117: 143-149.

Kameya,Y., H.Kanzaki, S.Toki and T.Abe (1989) Transfer of radish (Raphanus sativus L.) chloroplast into cabbage (Brassica oleracea L.) by protoplast fusion. Jap. J. Genet. 64: 27-34.

Koch,M., B.Haubold and T.Mitchell-Olds (2001) Molecular systematics of the Brassicaceae: evidence from coding plastidic matK and nuclear CHS sequences. Am. J. Bot. 88: 534-544.

Liere,K. and G.Link (1995) RNA-binding activity of the matK protein encoded by the chloroplast trnK intron mustard (Sinapis alba L.) Nucleic Acids Res. 23: 917-921.

Makaroff,C.A., I.J.Apel and J.D.Palmer (1989) The atp6 coding region has been disrupted and a novel reading frame generated in the mitochondrial genome of cytoplasmic male-sterile radish. Jour. Biol. Chem. 264: 11706-11713.

Makaroff,C.A., I.J.Apel and J.D.Palmer (1991) The role of cox1assoiciated repeated sequences in plant mitochondrial DNA rearrangements and radish cytoplasmic male sterility. Curr. Genet. 19: 183-190.

Motegi,T. (1999) Stoichiometric shift of mitochondria and chloroplast genomes, and the possibility of genetic induction of the shift by artificial chimera synthesis. Tokyo University of Agriculture and Technology Master thesis. 128 p.

Motegi,T., I.S.Nou, J.Zhou, A.Kanno, T.Kameya and Y.Hirata (2003) Obtaining an ogura-type CMS line from asymmetrical protoplast fusion between cabbage (fertile) and radish (fertile). Euphytica 129: 319-323. 
Neuhaus,H. and G.Link (1987) The chloroplast tRNAlys (UUU) gene from mustard (Sinapis alba) contains Class II intron potentially coding for a maturase-related polypeptide. Curr. Genet. 11: 251-257.

Noguchi,T., Y.Hirata and N.Yagishita (1992) Intervarietal and interspecific chimera formation by in vitro graft-culture method in Brassica. Theor. Appl. Genet. 83: 727-732.

Noguchi,T. and Y.Hirata. (1994) Vegetative and floral characteristics of interspecific Brassica chimeras produced by in vitro grafting. Euphytica 73: 273-280.

Ogura,H. (1968) Studies on the new male sterility in Japanese radish, with special reference to the utilization of this sterility towards practical raising of hybrid seeds. Mem. Fac. Agric. Kagoshima Univ. 6: 39-78.

Ohkawa,Y. (1986) Comparison of B. napus to B. campestris and $B$. oleracea based on the cytoplasmic characters; cytoplasmic male sterility and chloroplast DNA. JARQ. 19: 244-257.

Ostheimer, G.J., R.Wiliams-Carrier, S.Belcher, E.Osborne, J.Gierke and A.Barkman (2003) Group II intron splicing factors derived by diversification of an ancient RNA-binding domain. EMBO. J. 22: 3919-3929.

Soltis,D.E., R.K.Kuzoff, E.Conti, R.Gornall and K.Ferguson (1996)
MatK and $r b c L$ gene sequence data indicate that Saxifraga (Saxifragaceae) is polyphyletic. Am. J. Bot. 83: 371-382.

Steele,K.P. and R.Vilgalys (1994) Phylogenetic analyses of Polemoniaceae using nucleotide sequences of the plastid gene matK. Systematic Botany 19: 126-142.

Tateishi, A., H.Inoue, H.Shiba and S.Yamaki (2001) Molecular cloning of $\beta$-galactosidase from Japanese Pear (Pyrus pyrifolia) and its gene expression with fruit ripening. Plant Cell Physiol. 42: 492-498.

Tateishi, A., H.Inoue, H.Shiba and S. Yamaki (2002) Cloning and expression of $\beta$-galactosidase cDNA related to softening of avocado (Persea americana). J. Japan. Soc. Hort. Sci. 71: 48-55.

Wu, S., Y.Ueda, H.He, S.Nishihara and S.Matsumoto (2000) Phylogenetic analysis of Japanese Rosa species using matK sequences. Breed. Sci. 50: 275-281.

Xiang,Q.Y., D.E. Soltis and P.S.Soltis (1998) Phylogenetic relationships of Cornaceae and close relatives inferred from $m a t K$ and $r b c L$ sequences. Am. J. Bot. 85: 285-297.

Yamagishi,H. and T.Terachi (2001) Intra and inter-specific variation in the mitochondrial gene orf 138 of Ogura-type male sterile cytoplasm from Raphanus sativus and Raphanus raphanistrum. Theor. Appl. Genet. 103: 725-732. 